\title{
SCRaMbLE does the yeast genome shuffle
}

Synthetic yeast chromosomes can be radically rearranged using a method named SCRaMbLE (synthetic chromosome rearrangement and modification by loxPmediated evolution $)^{1}$. First published seven years ago, SCRaMbLE was devised by the yeast synthetic genome project (dubbed Sc2.0) as a way to enable deletion of unneeded genes once the Sc2.0 genome is finished, and eventually to engineer a synthetic yeast minimal genome. Now-well before the yeast synthetic genome itself is complete-the utility of this versatile, unbiased genome diversification method is showcased in a collection of articles in Nature Communications ${ }^{2-8}$, reporting a variety of applications, from generation of improved industrial yeasts to boosted production from engineered pathways.

DNA sequencing technologies can reliably read whole genomes, but approaches to decipher the biology of whole genomes are only now becoming available. Synthetic genomics-building genomes from scratch-is one way of understanding how a whole genome functions. Synthetic bacterial genomes came first, courtesy of J. Craig Venter and colleagues ${ }^{9}$, but, more recently, genome engineers have set their sights on Saccharomyces cerevisiae, a single-celled eukaryote used by molecular biologists, metabolic engineers and brewers worldwide.

The synthetic yeast genome project aims to replace native sequences of brewer's yeast with designed sequences. Sc2.0 isn't a frame-by-frame remake of the brewer's yeast genome. Rather, the design is bespoke, intricate and innovative. Repetitive regions and most introns are removed. Destabilizing regions, such as transposons, are eliminated. TAG stop codons are recoded to TAA to allow insertion of non-canonical amino acids. tRNA genes, which are hotspots for genome instability, are moved to a neochromosome. And bidirectional loxPsym sites are inserted in the $3^{\prime}$ UTR of all non-essential genes to enable 'SCRaMbLE-ing' of the genome (deletions, insertions and inversions), using an inducible Cre recombinase ${ }^{10}$. Induction of Cre with $\beta$-estradiol can be stopped by heat treatment.

The goal of Sc2.0 is a fully synthetic yeast with similar fitness as wild-type yeast and bearing 5,000 loxPsym sites. Six synthetic chromosomes have been completed to date, with ten left to recode ${ }^{10}$. The veracity of

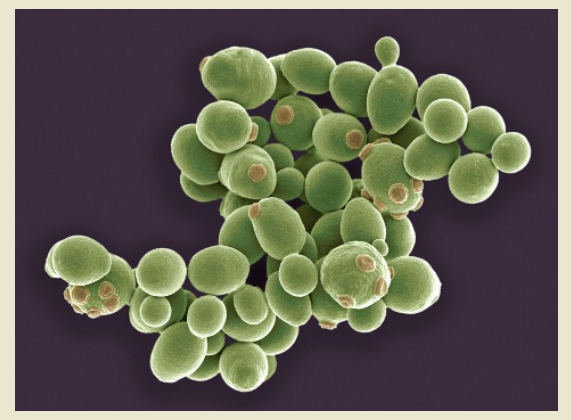

reintroducing them into a cell and reading out a phenotype.

Other applications involve nuanced SCRaMbLE screening strategies. For example, ReSCuES (reporter of SCRaMbLEd cells using efficient selection) from Luo et al exploits two auxotrophic markers; the first is functional only before SCRaMbLE, and the second only after SCRaMbLE, thereby establishing precise selection of SCRaMbLEd cells ${ }^{7}$. Finally, Hochrein et al. ${ }^{8}$ engineer a lightcontrolled Cre (L-SCRaMbLE), which has the benefit of rapid and completely reversible SCRaMbLE-ing.

SCRaMbLE as a tool for rapid genotype diversification has been established by sequencing the genomes of 64 SCRaMbLE'd strains ${ }^{11}$. In future work, Sc2.0 will apply the method to investigate mechanisms of speciation, minimal genome requirements and genome evolution in yeast.

Aside from these esoteric studies, SCRaMbLE should facilitate diverse applications of benefit to academic and industrial researchers alike, as the new collection of papers has shown ${ }^{2-8}$. Blount et al. ${ }^{2}$ rapidly shuffle the genome of a yeast harboring a synthetic version of chromosome V. In just a couple of weeks, they generate and sequence chromosomal rearrangements that ramp up production of plasmid-borne violacein or penicillin, and improve growth on xylose. By coupling SCRaMbLE with long-read nanopore sequencing, the authors present a fast route to identification of gene duplication, loss or repositioning that enables higher yields.

Wholesale reshuffling of an Sc2.0 genome is intriguing, but what about the less-wellcharacterized industrial yeasts? By mating haploid Sc2.0 strains with haploid wild-type Saccharomyces cerevisiae strains, Jia et al. ${ }^{3}$ and Shen et al. ${ }^{4}$ crucially show not only that synthetic Sc2.0 chromosomes function in heterozygous diploid industrial yeasts but that SCRaMbLE-ing produces diploid strains with improved industrial phenotypes, such as better growth at high temperatures. Using MuSIC (multiplexed SCRaMbLE iterative cycling) in their heterozygous diploid yeast, Jia et al. ${ }^{3}$ also find that multiple SCRaMbLE cycles diversify genotypes further and massively increase the yields of a pigmented product.

Taking aim at a far smaller target than an entire chromosome, Wu et al..$^{5}$ and Liu et al. ${ }^{6}$ apply SCRaMbLE to a pathway or set of genes in a test tube. Using SCRaMbLE like an enzyme, they rearrange genetic parts before
SCRaMbLE enables profound alterations in pathways, chromosomes or whole genomes that might otherwise never arise using alternative methods. It is conceivable that application of this unconventional genome shuffling technology will reveal the ways that large-scale changes to genomes can be used to benefit biotechnology. According to Hal Alper (University of Texas, Austin, uninvolved with the studies), "This suite of papers empowers the field with a newfound potential to reconfigure the yeast genome. Genome arrangement and organization is no longer a static, predetermined feature of cells, but can be viewed as a tunable design variable. Reconfigured chromosomes can provide valuable insights into genome architecture and function."

Innovations in the design of Sc2.0, the first eukaryotic synthetic genome, should also inform the human genome project write (HGP-write) as it endeavors to build a synthetic version of the human genome.

Susan Jones, Senior Editor

1. Dymond, J.S. et al. Nature 477, 471-476 (2011). org/10.1038/s41467-018-03143-w (2018).

3. Jia, B. et al. Nat. Commun. http://doi.org/10.1038/ s41467-018-03084-4 (2018).

4. Shen, M.J. et al. Nat. Commun. http://doi. org/10.1038/s41467-018-04157-0 (2018).

5. Wu, Y. et al. Nat. Commun. http://doi.org/10.1038/ s41467-018-03743-6 (2018).

6. Liu, W. et al. Nat. Commun. http://doi.org/10.1038/ s41467-018-04254-0 (2018).

7. Luo, Z. et al. Nat. Commun. http://doi.org/10.1038/ s41467-017-00806-y (2018).

8. Hochrein, L. et al. Nat. Commun. http://doi. org/10.1038/s41467-017-02208-6 (2018).

9. Gibson, D.G. et al. Science 319, 1215-1220 (2008).

10. Kannan, K. \& Gibson, D.G. Science 355 1024-1025 (2017).

11. Shen, Y. et al. Genome Res. 26, 36-49 (2016).
2. Blount, B.A. et al. Nat. Commun. http://doi. 